C-A/AP/\#231

March 2006

\title{
Warm Calibration Measurements of the 1.3 GHz Electron Gun
}

\author{
H. Hahn
}

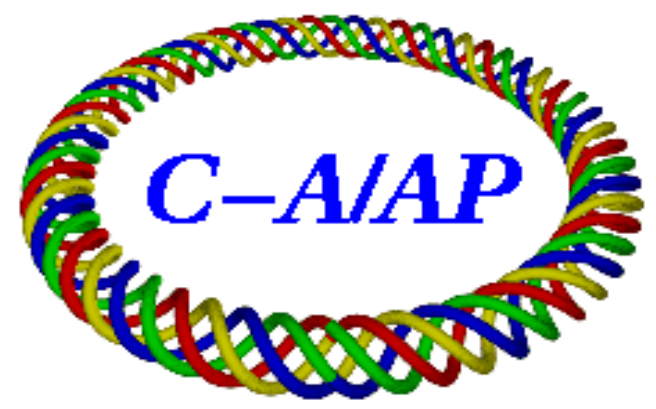

Collider-Accelerator Department Brookhaven National Laboratory Upton, NY 11973 
H. Hahn

March 10, 2006

\section{CALIBRATION MEASUREMENTS of the $1.3 \mathrm{GHz}$ ELECTRON GUN}

\section{Introduction}

The ongoing ERL research effort directed at e-cooling of RHIC beams requires a highbrightness high-current electron gun [1] While different approaches to achieving the requirements exist, the Brookhaven solution is based on a high-Q superconducting cavity. A $700 \mathrm{MHz}$ superconducting electron injector is being constructed by the Advanced Energy Systems company and will be delivered in 2007. Awaiting its availability, 1.3 $\mathrm{GHz} \mathrm{Nb}$ cavities shown in Fig. 1 are used for all relevant studies, such as properties of the superconductor and the semiconductor photocathode [2].

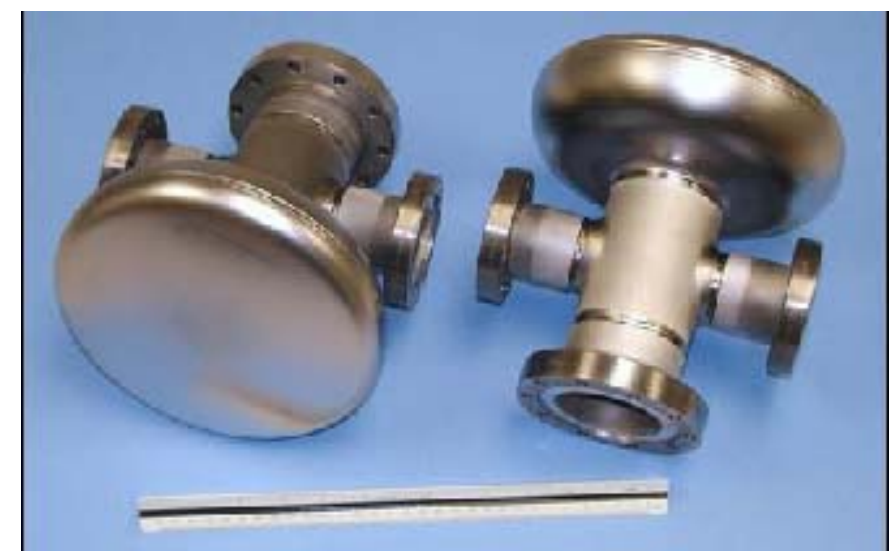

Fig. 1. 1.3 GHz Nb Cavities

The cavities are fabricated entirely from $\mathrm{Nb}$ [3]. Each cavity has a single beam pipe port and two additional coupler ports for the feed and pick-up coupler on the beam pipe. Accurate results for the electric field at operating conditions depends on the calibration of the pick-up probe. This calibration was done previously by a low-level room temperature measurement of the power delivered into the cavity and the power transmitted through the pick-up coupler. One of the two cavities was modified by adding a photocathode deposition stub as shown in Fig. 2.

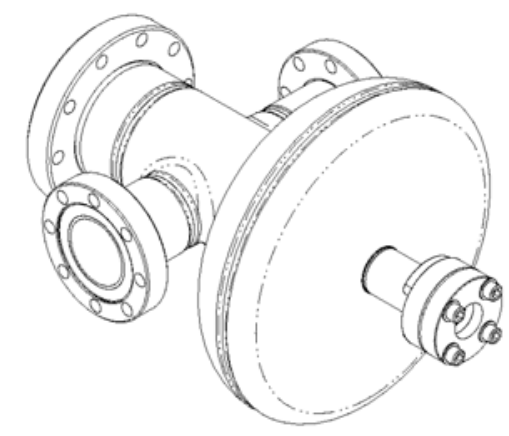

Fig. 2. Cavity with added choke joint. 
This provided the opportunity for a pick-up coupler calibration with significantly increased accuracy presented in this report. By feeding the cavity through this port one can achieve a fully matched condition without field distortion in the other port region. The coupling strength of the pick-up probe follows from a direct S21 transmission measurement with a network analyzer. This method and the calibration results are the topic of the note.

\section{Warm Measurements}

The measurements were performed using a $50 \Omega$ coaxial cable entering the cavity through the choke joint. The solid copper coax has a $1 \mathrm{~cm}$ O.D. to simulate the choke joint geometry and the center conductor protrudes the cable by $\sim 1 \mathrm{~cm}$. The penetration into the cavity is adjustable and used to achieve critical coupling. The input coupling strength has now been determined by also using the S11 phase method as described in the section below. The results presented here were obtained with recalibrated network analyzer $8753 \mathrm{C}$ which took out the losses in the instrument cables.

\section{Cavity}

The cavity resonance frequency is found from the minimum in the $\mathrm{S} 11$ amplitude to be

$$
f_{0}=1,295.74 \mathrm{MHz}
$$

with

$$
S_{11 d B}=-52 \mathrm{~dB} \text { or } S_{21}=2.5 \times 10^{-3} \text {. }
$$

The phase response indicates an under-coupled case, so that the coaxial cable coupling parameter is

$$
\beta_{C}=\frac{1-S_{11}}{1+S_{11}}=\frac{1-0.0025}{1+0.0025} \approx 0.995
$$

Alternatively, the relevant data for the reflection scattering coefficient from the cavity input is found from the phase difference between the two extrema,

$$
\Phi=167.8 / 2=83.8^{\circ}
$$

leading, in remarkable agreement, to the coupling

$$
\beta_{C}=\frac{\tan \Phi}{\sqrt{1+\tan ^{2} \Phi}}=0.994
$$

\section{Feed Coupler}

The loaded $Q_{L}$ and then, together with the $\beta_{C}$, the internal quality factor $Q_{0}$ of the cavity is obtained from the forward transmission coefficient $S_{21}$ between input and the "Feed" coupler. "Feed" coupler refers to the input coupler when the cavity is in operation.

The $3 \mathrm{~dB}$ bandwidth is measured as

$$
2 \Delta f_{3 d B}=0.384 \mathrm{MHz}( \pm 0.5 \%) \text {, }
$$

resulting in $Q_{L}=3374$ and the internal

$$
Q_{0}=\left(1+\beta_{C}+\beta_{F}+\beta_{P U}\right) Q_{L}=6728
$$


since $\beta_{F}$ and $\beta_{P U}$ can here be neglected.

The external $Q_{E}$ of the feed coupler is obtained from the $S_{21}$ measurement

$$
S_{21 d B}=-44.1 \pm 0.2 \mathrm{~dB} \text { or } S_{21}=6.24 \times 10^{-3}
$$

From the definition for the external coupling coefficient

$$
\beta_{F}=\frac{P_{F}}{P_{C}}=\frac{S_{21}^{2}}{1-S_{11}^{2}}=\frac{38.9 \times 10^{-6}}{1.00}=38.9 \times 10^{-6}
$$

The external $Q$ of the feed coupler now follows as

$$
Q_{F}=\frac{Q_{0}}{\beta_{F}}=1.73 \times 10^{8}
$$

\section{Pick up Coupler}

Same procedure (with average over 16 sweeps, $30 \mathrm{~Hz}$ IF, and smoothing over $10 \mathrm{kHz}$ ) yields for the pick-up coupler

$$
S_{21 d B}=-75 \pm 0.2 \mathrm{~dB} \text { or } S_{21}=1.78 \times 10^{-4} \text {, and } \beta_{P U}=3.16 \times 10^{-8} \text {. }
$$

The pick-up external quality factor is now

$$
Q_{P U}=2.13 \times 10^{11}
$$

\section{Cross Coupling}

Feed coupler and pick-up coupler are both connected to the single beam tube in this cavity and cross coupling is a concern [4]. The S21 measurement from feed to pick-up coupler show no measurable signal.

\section{$2^{\text {nd }}$ Feed Coupler}

Feed coupler modified by Kneisel measured in the same cavity.

$$
\begin{aligned}
& f_{0}=1295.81 \mathrm{MHz}, 2 \Delta f_{3 d B}=384.3 \\
& S_{21 d B}=-55.94 \text { or } S_{21}=1.60 \times 10^{-3} \quad(10 \mathrm{kHz} \text { smoothing }) \\
& Q_{F 2}=2.64 \times 10^{9}
\end{aligned}
$$

\section{$2^{\text {nd }}$ Pick up Coupler}

and the associated modified probe

$$
\begin{aligned}
& S_{21 d B}=-62.3 \mathrm{~dB} \text { or } S_{21}=7.64 \times 10^{-4}(20 \mathrm{kHz} \text { smoothing }) \\
& Q_{P U 2}=1.15 \times 10^{10}
\end{aligned}
$$

\section{Projected low-temperature measurements}

During low-temperature operation, the cavity is powered via the Feed coupler, which has the known temperature-independent external $Q_{F}$ from the warm measurement. A network analyzer provides a low-level loaded quality factor $Q_{L}$ from the $S_{21}$ between feed and pick-up. The internal $\mathrm{Q}$ is found from

$$
\frac{1}{Q_{0}}=\frac{1}{Q_{L}}-\frac{1}{Q_{F}}-\frac{1}{Q_{P U}} \approx \frac{1}{Q_{L}}-\frac{1}{Q_{F}}
$$


For electric field measurement at high power, the power source is connected to the cavity using a directional coupler with which the incoming power $P_{E}$ (and reflected power) is measured. From this measurement, the operational coupling coefficient $\beta_{F}^{\prime}$ at cold temperature (different from the warm $\beta_{F}$ ) is determined, and the power dissipated in the cavity is then given by

$$
P_{C}=\frac{4 \beta_{F}^{\prime}}{\left(1+\beta_{F}^{\prime}\right)^{2}} P_{F}
$$

A low level $S_{11}$ now provides the coupling factor at low field, $\beta_{F}^{\prime}$ which can serve as verification of the directional coupler result for the power dissipated in the cavity. Any difference is attributable to field emission or multipacting.

Superfish simulations provide the relationship between peak electric field on the cathode and the power dissipated in the cavity, (courtesy Y. Zhao)

$$
E_{0}=1.59 \times 10^{6} \sqrt{P_{C}}[\mathrm{~V} / \mathrm{m}]
$$

Alternatively, the electric field can be obtained from a power measurement at the pick-up coupler, $P_{P U}$ according to

$$
E_{0}=1.59 \times 10^{6} \sqrt{\frac{Q_{P U}}{Q_{F}} P_{P U}}=55.8 \times 10^{6} \sqrt{P_{P U}}
$$

Although the signal level at the pick-up is about three orders of magnitude smaller than the feed signal, it provides a correct measurement of the electric field even in the presence of other losses due to field emission or multipacting.

\section{Coupling coefficient from S11 measurement}

The $S_{11}$ reflection coefficient of a resonate cavity with unloaded $Q_{0}$ at the resonance $f_{0}$ and driven by the power source with is given by the generalized expression

$$
S_{11}=\frac{\beta-(1-j \delta)}{\beta+(1+j \delta)}
$$

with the coupling parameter $\beta$ and the frequency shift from the resonance, normalized to the half-3dB bandwidth of the unloaded cavity,

$$
\delta=\frac{\Delta f}{(\Delta f)_{3 d B}}
$$

where

$$
(\Delta f)_{3 d B}=\frac{f_{0}}{2 Q_{0}}
$$

Assuming an under-coupled driven cavity, the coupling parameter is found from the

minimum of the amplitude of $S_{11}$ at the resonance, 


$$
\beta=\frac{1-\left(S_{11}\right)_{f_{0}}}{1+\left(S_{11}\right)_{f_{0}}}
$$

Alternatively, and better for a close to critically coupled cavity, the coupling parameter can be found from the phase response of the reflection,

$$
\varphi=\operatorname{atan} \frac{2 \beta \delta}{1-\beta^{2}+\delta^{2}}
$$

The phase in vicinity of the resonance is almost $\mathrm{S}$-shaped with easily determined extrema of the phase $\Phi$ at $\delta_{\Phi}= \pm \sqrt{1-\beta^{2}}$. The value of the phase extremum is independent of the quality factor and is found to be

$$
\Phi=\operatorname{atan}\left(\frac{\beta}{\sqrt{1-\beta^{2}}}\right)
$$

which is measured in the network analyzer with great accuracy. The coupling parameter now follows from

$$
\beta=\frac{\tan \Phi}{\sqrt{1+\tan ^{2} \Phi}}
$$

\section{Acknowledgements}

The need for the calibration measurements were pointed out and the measuring procedure was suggested by Ilan Ben-Zvi. The Superfish computations were made by Y. Zhao. The adjustable coax-cable probes were provided by R. Sikora from the C-A Instrumentation Division.

\section{References}

1. I. Ben-Zvi et al., Proc. PAC 2005, p.2741

2. T. Rao et al., Proc. PAC 2005, p.2556

3. M. Cole et al., Proc. PAC 2005, p.3514

4. Y. Zhao and M. Cole, Proc PAC 2003, p. 2050 